\title{
Left Ventricular Pseudoaneurysm Caused by a Left Ventricular Venting Catheter via the Right Superior Pulmonary Vein during Thoracic Aortic Surgery: A Case Report
}

\author{
Taiju Watanabe*, Tetsuya Yoshida \\ Department of Cardiovascular Surgery, Hokushin General Hospital, Nagano, Japan \\ Email: ^twatanabe.cvsg@tmd.ac.jp
}

How to cite this paper: Watanabe, T. and Yoshida, T. (2019) Left Ventricular Pseudoaneurysm Caused by a Left Ventricular Venting Catheter via the Right Superior Pulmonary Vein during Thoracic Aortic Surgery: A Case Report. World Journal of Cardiovascular Surgery, 9, 41-46.

https://doi.org/10.4236/wjcs.2019.94005

Received: March 11, 2019

Accepted: April 19, 2019

Published: April 22, 2019

Copyright $\odot 2019$ by author(s) and Scientific Research Publishing Inc. This work is licensed under the Creative Commons Attribution International License (CC BY 4.0).

http://creativecommons.org/licenses/by/4.0/

\begin{abstract}
A left ventricular (LV) pseudoaneurysm is one of the complications of acute myocardial infarction. It is also reported after chest trauma, cardiac surgery, and endocarditis. We report a rare case of an LV pseudoaneurysm induced by an $\mathrm{LV}$ venting catheter through the right superior pulmonary vein during thoracic aortic surgery. A 77-year-old man was referred for surgical repair of a distal aortic arch aneurysm. He underwent total aortic arch reconstruction with the frozen elephant trunk technique. The early postoperative period was uneventful. Postoperative contrast computed tomography and transthoracic echocardiography (TTE) revealed a pseudoaneurysm with a narrow neck at the apex of the LV that had sub-clinically progressed. Because of the risk of spontaneous rupture, an urgent aneurysmectomy was performed via a repeat sternotomy. Under cardioplegic arrest, the pseudoaneurysm was opened and the small orifice, which communicated with the LV, was confirmed. No myocardial ischemic changes were observed around the orifice. The pseudoaneurysm was thought to be induced by endocardial laceration by the tip of the venting catheter. The pseudoaneurysm was closed by linear repair reinforced with felt strips. The patient recovered well and was discharged 18 days after the second surgery. TTE showed no recurrence of LV aneurysm at the last follow-up.
\end{abstract}

\section{Keywords}

Left Ventricular Pseudoaneurysm, Left Ventricular Venting Catheter, Right Superior Pulmonary Vein

\section{Introduction}

A left ventricular (LV) pseudoaneurysm is one of the complications of acute 
myocardial infarction. It is also reported after chest trauma, cardiac surgery, and endocarditis [1] [2]. Congestive heart failure, chest pain, and dyspnea are the most frequently reported symptoms, but $>10 \%$ of patients are asymptomatic. Early surgical repair is recommended, because there is potential for spontaneous rupture [3]. An LV venting technique is frequently used to provide a dry operative field during valvular surgery or to prevent ventricular distention during systemic cooling. There are some reports of LV pseudoaneurysm resulting from apical venting [4] [5] [6] [7], but LV pseudoaneurysm due to an LV venting catheter inserted from the right superior pulmonary vein has rarely been reported. We present a rare case of LV pseudoaneurysm induced by an LV venting catheter via the right superior pulmonary vein during thoracic aortic surgery. Written informed consent was obtained from the patient for publication of this case report and accompanying images.

\section{Case}

A 77 year-old man was referred for surgical repair of a distal aortic arch aneurysm. His past medical history included long-standing hypertension and diabetes mellitus. Contrast computed tomography (CT) showed a distal aortic arch aneurysm $66 \mathrm{~mm}$ in diameter and an infra-renal abdominal aortic aneurysm 44 $\mathrm{mm}$ in diameter (Figure 1). Transthoracic echocardiography (TTE) showed good LV ejection fraction without wall motion abnormality or valvular disease. Coronary angiography revealed no significant stenosis.

The patient underwent total aortic arch reconstruction with the frozen elephant trunk (FET) technique. Cardiopulmonary bypass (CPB) was established with ascending aorta and right atrium cannulation. An LV venting catheter was inserted via the right superior pulmonary vein. Under moderately hypothermic circulatory arrest with antegrade cerebral perfusion, the distal part of the ascending aorta was transected, and FET was advanced through the aortic arch into the proximal descending aorta, and deployed. Thereafter, a four-branched graft was anastomosed to the stump of the aortic arch with anterior translocation. Intraoperative hemostasis after the discontinuation of CPB was per usual. The early postoperative period was uneventful. Routine TTE, performed 8 days post-surgery, showed preserved LV function and no unusual findings were detected.

Routine contrast CT, performed 15 days post-surgery, detected a pseudoaneurysm with a narrow neck at the apex of the LV (Figure 2). TTE, performed 16 days post-surgery, confirmed the presence of the pseudoaneurysm with a diameter of $35 \times 26 \mathrm{~mm}$ at the apex of the LV, communicating with the LV through an orifice $8 \mathrm{~mm}$ in diameter (Figure 3). There were no findings of perioperative myocardial infarction such as electrocardiogram changes, cardiac enzyme elevation, or regional wall motion abnormality around the pseudoaneurysm. The pseudoaneurysm was thought to be induced by endocardial laceration by the tip of the venting catheter. The pseudoaneurysm had subclinically pro- 
gressed in one week. Because of the risk of spontaneous rupture, an urgent aneurysmectomy was performed via a repeat sternotomy with femoro-femoral bypass. Mediastinal and pericardial adhesions were dissected and the pseudoaneurysm at the LV apex was approached. Under cardioplegic arrest, the pseudoaneurysm was opened and the small orifice, which communicated with the LV, was confirmed (Figure 4). There was no thrombus formation in the pseudoaneurysm. No myocardial ischemic changes were observed around the orifice. The pseudoaneurysm was closed by linear repair reinforced with felt strips. The postoperative period was uneventful. The patient was discharged 18 days after the second surgery. At the last follow-up visit, six months after the second surgery, TTE showed no recurrence of aneurysm (Figure 5).
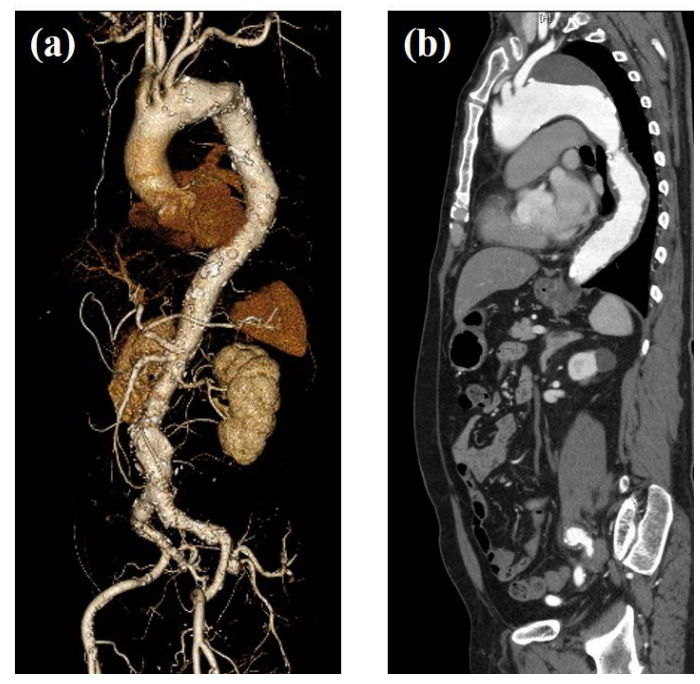

Figure 1. Preoperative contrast computed tomography (CT) revealed a distal aortic arch aneurysm $66 \mathrm{~mm}$ in diameter, and an infrarenal abdominal aortic aneurysm $44 \mathrm{~mm}$ in diameter; (a) three-dimensional view (b) sagittal view.

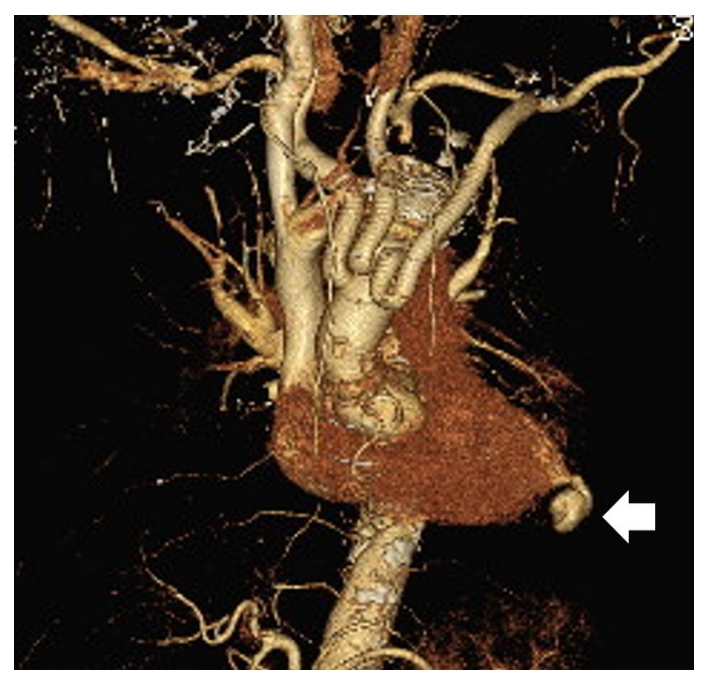

Figure 2. Postoperative contrast CT revealed a pseudo aneurysmal formation at the apex of the left ventricle (LV) with a narrow neck (arrow). 

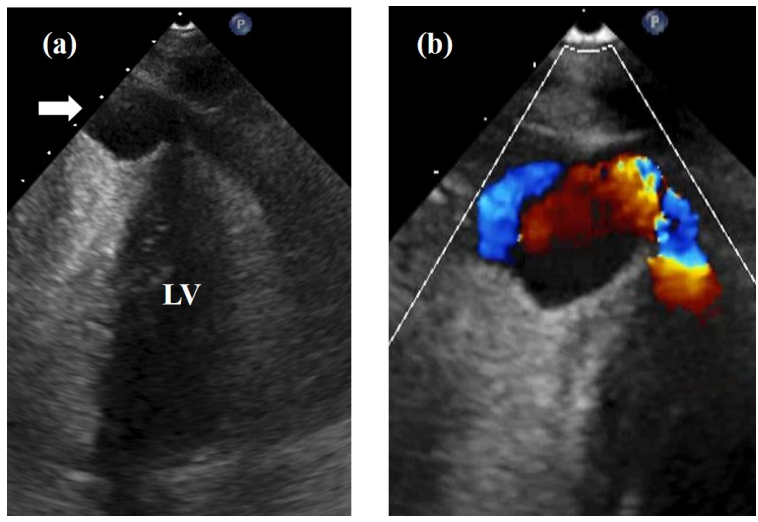

Figure 3. Postoperative transthoracic echocardiography (TTE) revealed a cavity $35 \times 26 \mathrm{~mm}$ in size communicating with the LV ((a) arrow). Color Doppler flow mapping identified an inflow into the cavity from the LV (b). LV; left ventricle.

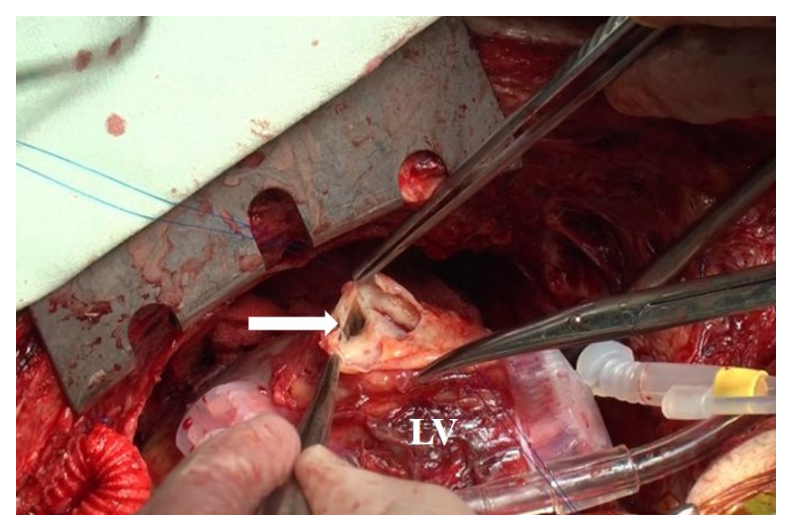

Figure 4. The pseudoaneurysm at the apex of LV was opened under cardioplegic arrest. The orifice into the LV was identified (arrow), and no thrombus formation was observed in the pseudoaneurysm. No myocardial ischemic changes were observed around the orifice. LV; left ventricle.

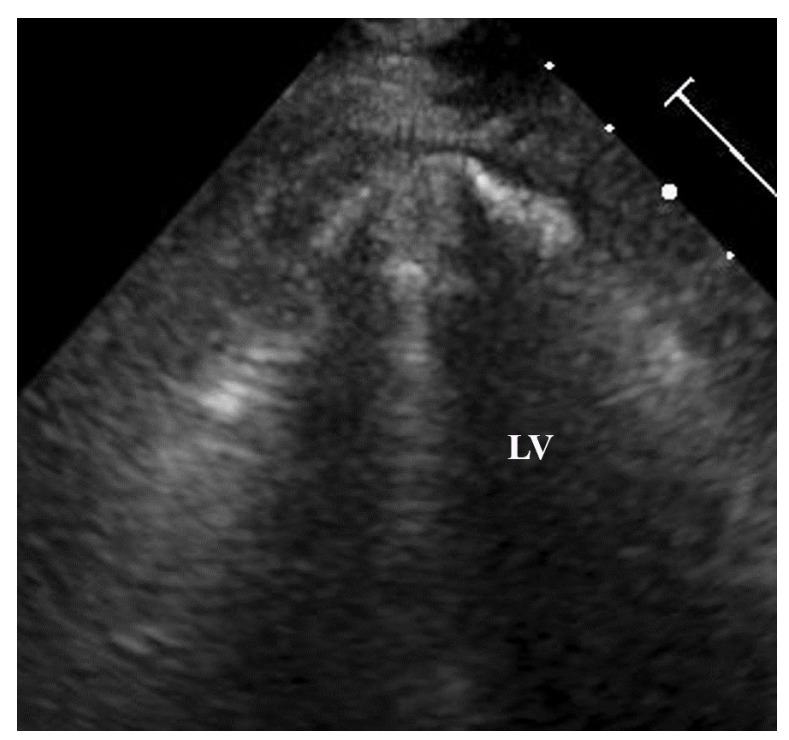

Figure 5. Follow-up transthoracic echocardiography (TTE) showed no recurrence of aneurysm at the apex of the LV. LV; left ventricle. 


\section{Discussion}

Pseudoaneurysms in the LV usually occur when the myocardial rupture following myocardial infarction is sealed by pericardium and fibrous tissue. Most LV pseudoaneurysms are related to myocardial infarction but some cases occur as a complication of cardiac surgery, mainly secondary to mitral valve surgery and aneurysmectomy. As soon as a pseudoaneurysm is detected, it should be surgically repaired to avoid spontaneous rupture.

Few cases of LV pseudoaneurysm related to an LV venting catheter via the right superior pulmonary vein have been reported in the literature [8] [9] [10]. These pseudoaneurysms progress without symptoms and the duration from the first surgery to the diagnosis varies. Hong and colleagues reported the first case of a pseudoaneurysm due to a venting catheter inserted from the right superior pulmonary vein during surgery for a type A aortic dissection but that case was managed conservatively because of the patient's refusal to undergo resurgery [8]. Ryomoto et al. also reported a case of LV pseudoaneurysm related to a venting catheter following mitral valve replacement with bioprosthesis. Although a venting catheter was gently introduced through the implanted bioprosthesis under direct vision, the LV pseudoaneurysm was diagnosed three years after the surgery. The aneurysmectomy was performed via the fourth left intercostal space [9].

$\mathrm{LV}$ venting technique is one of the basic procedures during cardiac surgery, but it is associated with serious complications such as cardiac perforation and pseudoaneurysm. During the insertion of the LV venting catheter via the right superior pulmonary vein, the position of the tip of the catheter in the LV should be confirmed by transesophageal echocardiography (TEE). During hypothermia, as in the present case, stiffness of the CPB cannula is increased, and the LV venting catheter, in prolonged close contact with the endocardium, can cause lacerations leading to formation of an LV pseudoaneurysm [10].

Aneurysmectomy was essential but reoperation was more challenging due to mediastinal and pericardial adhesions. Rahim and colleagues reported a successful percutaneous procedure by delivering coils retrogradely in the neck of the LV pseudoaneurysm through the aortic valve under LV angiography [11]. In high-risk surgical patients, percutaneous closure of the LV pseudoaneurysm can be done.

\section{Conclusion}

We reported a rare case of an LV pseudoaneurysm induced by the LV venting catheter through the right superior pulmonary vein during thoracic aortic surgery. Great care should be taken during the placement of the LV venting catheter with confirmation of the position of the catheter by intraoperative TEE. If an LV pseudoaneurysm is diagnosed postoperatively, prompt surgical repair should be performed. 


\section{Conflicts of Interest}

The authors declare no conflicts of interest regarding the publication of this paper.

\section{References}

[1] Sutherland, G.R., Smyllie, J.H. and Roelandt, J.R. (1989) Advantages of Colour Flow Imaging in the Diagnosis of Left Ventricular Pseudoaneurysm. Heart, 61, 59-64. https://doi.org/10.1136/hrt.61.1.59

[2] Namboodiri, N., Dora, S.K., Thomas, B. and Misra, M. (2008) Subannular Left Ventricular Pseudoaneurysm Following Mitral Valve Replacement. Journal of Cardiothoracic Surgery, 3, 28. https://doi.org/10.1186/1749-8090-3-28

[3] Frances, C., Romero, A. and Grady, D. (1988) Left Ventricular Pseudoaneurysm. Journal of the American College of Cardiology, 32, 557-661. https://doi.org/10.1016/S0735-1097(98)00290-3

[4] Kao, C. L.and Chang, J.P. (2003) Left Ventricular Pseudoaneurysm Secondary to Left Ventricular Apical Venting. Texas Heart Institute Journal, 30, 162-163.

[5] Sá, M.I., Lli, W., Sheppard, M.N. and Kilner, P.J. (2008) Mycotic Left Ventricular False Aneurysm at the Site of an Apical Vent Presenting 24 Years after Aortic Valve Surgery. Circulation, 118, e501-e503. https://doi.org/10.1161/CIRCULATIONAHA.107.732115

[6] Bizzarri, F., Rose, D., Frati, G. and Muzzi, L. (2006) Left Ventricular Postoperative False Aneurysm Following Apical Venting. Journal of Cardiothoracic Surgery, 1, 41. https://doi.org/10.1186/1749-8090-1-41

[7] Lopes, R., Almeida, J., Silva, J.C., Almeida, P.B., Madureira, A.J., Ramos, I., et al. (2011) Spontaneous Closure of a Left Ventricle Pseudoaneurysm Following Apical Venting. European Journal of Echocardiography, 12, E6.

[8] Hong, J.H., Choi, J.W., Moon, J.H. and Lim, S.H. (2007) Pseudoaneurysm of Left Ventricular Apex Caused by a Left Ventricular Venting Catheter through the Right Superior Pulmonary Vein. The Journal of Thoracic and Cardiovascular Surgery, 134, 229-230. https://doi.org/10.1016/j.jtcvs.2007.01.018

[9] Ryomoto, M., Mitsuno, M., Fukui, S., Miyamoto, Y. and Hao, H. (2013) Subepicardial Aneurysm by Venting Catheter via the Right Superior Pulmonary Vein. General Thoracic and Cardiovascular Surgery, 61, 148-151. https://doi.org/10.1007/s11748-012-0118-Z

[10] Laguna, G., Echevarría, J.R. and Fernández, M. (2015). Left Ventricular Pseudoaneurysm Following Thoracic Aorta Replacement. Revista Española de Cardiología, 68, 340. https://doi.org/10.1016/j.recesp.2014.05.022

[11] Rahim, S.A., Greason, K.L., Bjarnason, H. and Rihal, C.S. (2009) Left Ventricular Pseudoaneurysm. Journal of the American College of Cardiology, 54, 740. https://doi.org/10.1016/j.jacc.2009.02.087 